\title{
AN APPROACH FOR ROUTINE ANALYTICAL DETECTION OF BEESWAX ADULTERATION USING FTIR-ATR SPECTROSCOPY
}

\author{
Lidija Svečnjak ${ }^{*}$ \\ Goran Baranović ${ }^{2}$ \\ Marko Vinceković ${ }^{3}$ \\ Saša Prđun ${ }^{1}$ \\ Dragan Bubalo' \\ Ivana Tlak Gajger ${ }^{4}$ \\ ${ }^{1}$ University of Zagreb Faculty of Agriculture, Department of Fisheries, \\ Apiculture and Special Zoology, 10000 Zagreb, Croatia \\ ${ }^{2}$ Rudjer Boskovic Institute, Division of Organic Chemistry and Biochemistry \\ (Laboratory of Molecular Spectroscopy), 10000 Zagreb, Croatia \\ 3University of Zagreb Faculty of Agriculture, Department of Chemistry, \\ 10000 Zagreb, Croatia \\ ${ }^{4}$ University of Zagreb, Faculty of Veterinary Medicine, \\ Department of Biology and Pathology of Fish and Bees, 10000 Zagreb, Croatia \\ *corresponding author: Isvecnjak@agr.hr \\ Received 8 October 2014; accepted 10 July 2015
}

\begin{abstract}
A b s tract
Although beeswax adulteration represents one of the main beeswax quality issues, there are still no internationally standardised analytical methods for routine quality control. The objective of this study was to establish an analytical procedure suitable for routine detection of beeswax adulteration using FTIR-ATR spectroscopy. For the purpose of this study, reference IR spectra of virgin beeswax, paraffin, and their mixtures containing different proportions of paraffin ( 5 - $95 \%$ ), were obtained. Mixtures were used for the establishment of calibration curves. To determine the prediction strength of IR spectral data for the share of paraffin in mixtures, the Partial Least Squares Regression method was used. The same procedure was conducted on beeswax-beef tallow mixtures. The model was validated using comb foundation samples of an unknown chemical background which had been collected from the international market $(n=56)$. Selected physico-chemical parameters were determined for comparison purposes. Results revealed a strong predictive power $\left(R^{2}=0.999\right)$ of IR spectra for the paraffin and beef tallow share in beeswax. The results also revealed that the majority of the analysed samples (89\%) were adulterated with paraffin; only 6 out of $56(11 \%)$ samples were identified as virgin beeswax, $28 \%$ of the samples exhibited a higher level of paraffin adulteration ( $>46 \%$ of paraffin), while the majority of the analysed samples (50\%) were found to be adulterated with $5-20 \%$ of paraffin. These results indicate an urgent need for routine beeswax authenticity control. In this study, we demonstrated that the analytical approach defining the standard curves for particular adulteration levels in beeswax, based on chemometric modelling of specific IR spectral region indicative for adulteration, enables reliable determination of the adulterant proportions in beeswax.
\end{abstract}

Keyword: adulterants share detection, beeswax adulteration, fingerprint region, FTIR-ATR spectroscopy.

\section{INTRODUCTION}

A specific lipid-based chemical composition of beeswax plays an important role in a honeybee colony as a source of nest-mate recognition cues and as construction material for honeycomb cells where honey and brood are being capped (Breed et al., 1995; Fröhlich et al., 2000; D'Ettorre et al., 2006; Buchwald et al., 2009). It is, therefore, important that the foundations on which the honeycombs will be built are genuine. 
Although beeswax adulteration has been one of the main beeswax quality issues for more than a decade, there are still no internationally standardised analytical methods for routine beeswax authenticity control (Bogdanov, 2004a). Nowadays, more than 15 different natural (petroleum-derived, mineral, animal, and plant waxes) and artificial/ industrial waxes can be used as beeswax adulterants (Bogdanov, 2009). Among them, the adulteration with paraffin waxes represents the greatest problem due to its wide availability, low price, and physico-chemical properties (a chemically inert, white or colourless and odourless substance) that altogether makes it "ideal" for adulteration.

The analytical methods defining pure-beeswaxcomposition criteria based on 10 classical physicochemical parameters determined in accordance with European Pharmacopoeia (Council of Europe, 2007) and the Methods of German Society for Fat Science (DGF-M-V-6, 1957), have been proposed for routine beeswax authenticity testing (Bogdanov, 2004b; 2009). However, as reported in the past and current literature, there are several factors affecting the proposed analytical range values for pure beeswax and detection of its adulteration (Tulloch, 1973; Bernal et al., 2005; Jiménez et al., 2007; Maia and Nunes, 2013). Along with possible deviations in proposed range values arising from the beeswax origin (geographical and/or race-related) (Beverly et al., 1995), a temperature treatment (an integral part of the production of comb foundation) can cause measurable changes of certain physico-chemical values (primarily acid and ester value). Consequently, particular beeswax samples can be displaced beyond the limits proposed for pure beeswax. These findings undermine the use of these measurements as reliable analytical tools for detection of beeswax adulteration.

Along with the mentioned classical analytical methods, very sophisticated analytical tools have been employed to investigate detailed beeswax chemical composition and/or to find more reliable analytical tools for detection of beeswax adulteration. The tools referred to are different techniques of high-temperature gas chromatography - mass spectrometry (Aichholz and Lorbeer, 1999, 2000; Jiménez et al., 2003; 2004; 2006; 2009; Serra Bonvehi and Orantes Bermejo, 2012; Maia and Nunes, 2013; Waś et al., 2014). These methods, though, have not yet met the analytical eligibility for routine beeswax control. The reason being, that the establishment of calibration curves and validation by comparing the results with other commonly used methods were not performed. Also, a general interpretation of beeswax IR spectrum and investigation of molecular structure and dynamics of beeswax by IR and Raman spectroscopy were reported in several spectroscopic studies (Birshtein and Tul'chinskii, 1977; Edwards et al., 1996; Zimnicka and Hacura, 2006; Muscat et. al., 2014).

There has been a recent report on the feasibility study of a single-reflection Attenuated Total Reflectance Fourier Transform Infrared Spectroscopy (FTIR-ATR) for detection of beeswax adulterants (Maia et al., 2013). This spectroscopic study revealed direct and reagent free method that provides good detection limits $(0.5-5 \%)$ of four of the most commonly employed beeswax adulterants: paraffin, microcrystalline wax, tallow, and stearic acid.

Considering the reported advantages of this spectroscopic technique and its detection limits for beeswax adulterants, the objective of this study was to establish an analytical procedure suitable for routine detection of beeswax adulteration by paraffin and beef tallow using the FTIR-ATR spectroscopy. The existing methodology was modified in terms of faster spectra acquisition, different data processing, and establishment of a calibration curve procedure. Also, the purpose of this work was to validate the method by comparing the results of the performed analytical procedure with those of commonly used physico-chemical analyses.

\section{MATERIAL AND METHODS}

\section{Samples}

To obtain the reference infrared (IR) spectra of pure beeswax, virgin beeswax samples (wild-built combs) were collected directly from the beehives maintained at experimental apiaries in different geographical regions across Croatia in 2013 - 2014 $(n=21)$. Furthermore, a total of 56 comb-foundation samples were collected from the beeswax foundation manufacturers and/or specialised beekeeping shops in 9 European countries (Austria, Bosnia and Herzegovina, Croatia, Germany, Hungary, Italy, Macedonia, Serbia, Slovenia; $n=52$ ) and in other countries (Russia, Argentina, China; $n=4$ ). Four paraffin samples with different melting points were obtained from Kemig (paraffinum solidum, Ph. Eur. 7.8, Croatia) and from the beekeepers (paraffin used for hive waxing). Beef tallow was obtained from the local butcher shop. Samples were analysed directly as obtained, without using purification or any other chemical reagents. Samples were stored in the dark at room temperature before analysis. 
Preparation of beeswax-paraffin and beeswaxbeef tallow mixtures for calibration (standard curves)

Mixtures of virgin beeswax containing different proportions (5 - 95\%) of paraffin wax were prepared. This procedure was performed to obtain reference samples exhibiting physico-chemical features and IR spectra specific for particular adulteration levels $(5,10,20,30,40,50,60,70,80,90,95 \%)$. These samples were used for the establishment of calibration (standard) curves exhibiting a relationship between the measurement response and the known concentrations of the analyte. Virgin beeswax (wild-built combs) collected from 7 hives, and Kemig paraffin wax (with a melting point of $56^{\circ} \mathrm{C}$ ) were used for the mixture preparation (w/w). Mixtures were homogenised by melting at $90^{\circ} \mathrm{C}$ for $3 \mathrm{~h}$ and re-solidified by allowing the mixture to cool to room temperature. The same analytical procedure was initially assayed for the virgin beeswax-beef tallow mixtures that were prepared in the same way in order to validate the procedure on another adulterant type.

\section{FTIR-ATR spectra acquisition and data processing}

The IR spectra of all sample types (virgin beeswax, paraffin, tallow, beeswax-paraffin, and beeswax-tallow mixtures) were recorded using an ABB Bomem MB102 FTIR (fourier-transform infrared) spectrometer with a DTCS (deuterated triglycine sulfate) detector and CsI (cesium iodide) optics. A singlereflection high temperature heated diamond ATR (Attenuated Total Reflectance) system (Golden Gate, Specac) and beam condensing optics made of ZnSe were used to acquire spectra. The depth of the beam penetration into the sample was around $2 \mu \mathrm{m}$. Spectra were recorded in a mid-infrared region (4000 - $400 \mathrm{~cm}^{-1}$ ) in accordance with the method described by Maia et al. (2013), including a few modifications regarding spectra acquisition and data processing. Prior to the acquisition of the spectra, each sample was left on the ATR plate for 3 minutes to allow melting, homogenisation, and stabilisation of the sample. Approximately $0.05 \mathrm{~g}$ of the sample was used to acquire the sample's IR spectrum. Two replicate spectra of each sample were recorded using the same aliquot, and 64 scans were collected for each spectrum. A new background spectrum was acquired before each sample. To exclude the noisy parts of the spectra, only the spectral range between 3700 and $600 \mathrm{~cm}^{-1}$ was taken into consideration for further data analysis. Raw IR spectra of the analysed samples were stored and pre-analysed using Origin version 8.1 (Origin Lab Corporation). Given that the most characteristic spectral features of pure beeswax appear in the spectral region between 1800 - $900 \mathrm{~cm}^{-1}$, this particular region, a fingerprint region, was used for further chemometric and statistical modelling. The use of the IR spectra of prepared beeswax-paraffin mixtures was carried out to generate the calibration curves covering the entire range $(0-100 \%)$ of paraffin adulteration. The overall fingerprint region was used for that purpose. The same procedure was performed on beeswaxbeef tallow mixtures.

Additionally, the IR spectra of microcrystalline wax, stearic acid, and carnauba wax were recorded. This was done to create a reference spectra collection for comparative qualitative detection of their potential presence in analysed comb foundation samples. Detection of other adulterants was investigated by comparing the analysed foundations to the IR spectra in available spectral databases (such as shellac wax and wool lanoline in the FTIR-ATR spectral database of the University of Tartu, Estonia, Institute of Chemistry).

To investigate the possible influence of a hightemperature treatment on the spectral features of pure beeswax, a virgin beeswax sample used in this study for mixtures preparation, was subjected to $125^{\circ} \mathrm{C}$ for 45 minutes (the most commonly used treatment in the comb foundation production). After cooling to room temperature, the sample's spectrum was recorded and compared to the one obtained before the exposure to $125^{\circ} \mathrm{C}$.

\section{Quality assessment of beeswax using the physico-chemical parameters}

Selected physico-chemical analyses, i.e. determination of acid number, ester number, ester/acid ratio, saponification number, and melting point, were performed according to European Pharmacopeia Commission (Council of Europe, 2007) in all samples. The relationship between the paraffin adulteration level and the obtained values of the physico-chemical parameters was, thus, assessed. The results of the physico-chemical parameters have been assessed according to the quality criteria proposed by International Honey Commission (Bogdanov, 2009).

\section{Statistical analysis}

Chemometric modelling and statistical analyses were performed using the statistical packages MatLab 7.11 2010b (MathWorks Inc.) and Statistica 7 (StatSoft Inc.). A factor-based Partial Least Squares Regression (PLSR) method was used to determine the prediction strength of FTIR-ATR spec- 
troscopy for the paraffin and tallow adulteration level using the sample set of prepared beeswax-paraffin and beeswax-tallow mixtures for calibration. The model was validated on a set of samples consisting of 56 comb foundations of unknown chemical background collected from the market. Model calibration performance and accuracy were evaluated using coefficient of determination $\left(R^{2}\right)$ and mean squared error (MSE) value. For comparison purposes, the relationship between the values of physicochemical parameters and the paraffin share was assessed on the mixtures using simple regression models. The paraffin share prediction performances of IR spectral data and physico-chemical measurands were also compared.

\section{RESULTS}

FTIR-ATR spectra of virgin beeswax, paraffin, and tallow

Results presented in Fig. 1a show the characteristic FTIR-ATR spectrum of genuine beeswax
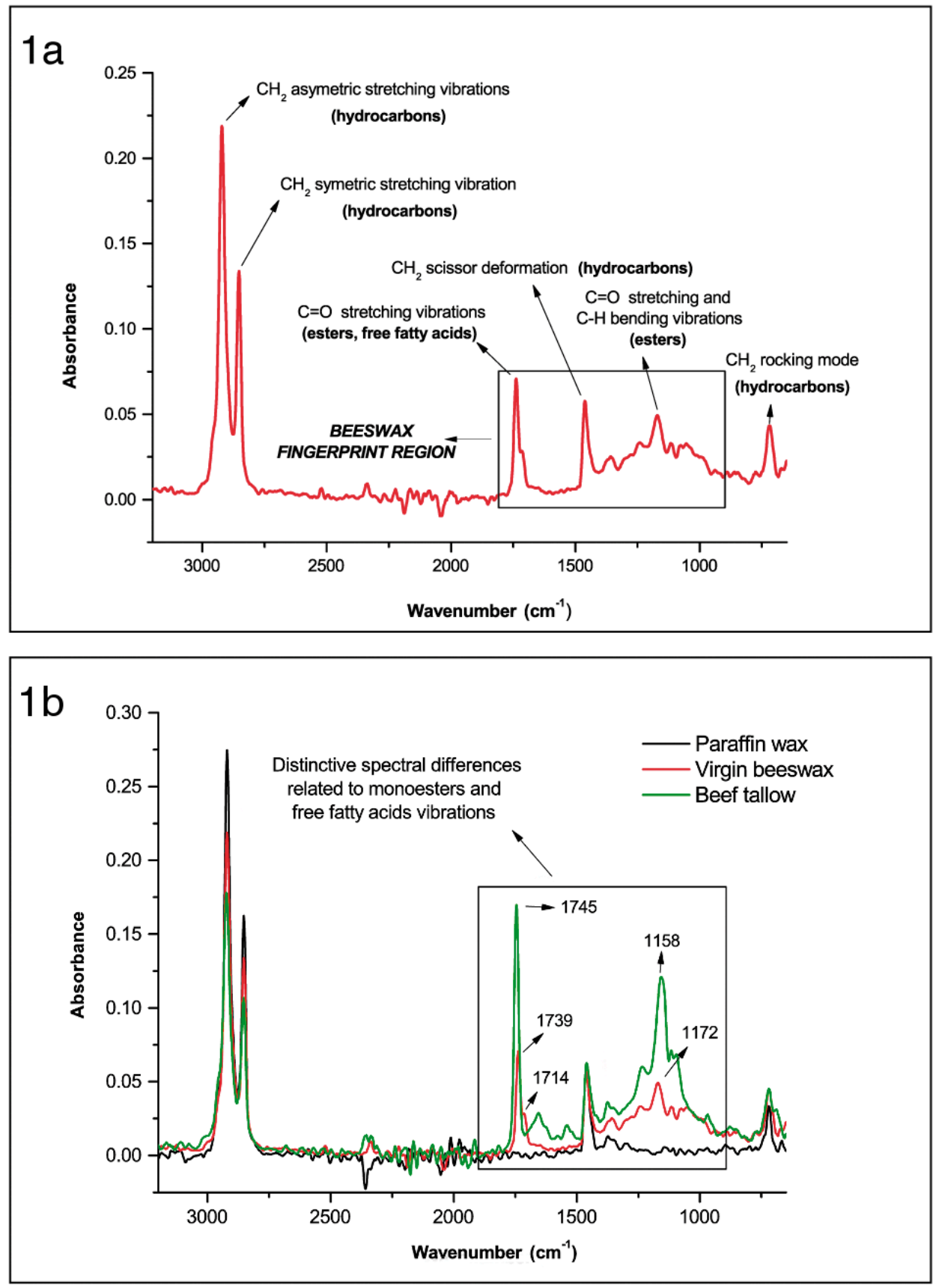

Fig. 1. (a) Characteristic FTIR-ATR spectrum of virgin beeswax with assignment of underlying absorption bands;

(b) Distinctive spectral differences between virgin beeswax, paraffin, and tallow occurring in the fingerprint region. 
and underlying molecular vibrations. Beeswax represents a complex organic mixture of numerous compounds. Yet, only the most dominating ones are observable in a typical IR spectrum of genuine beeswax; mostly analyte signals belonging to hydrocarbons, esters and free fatty acids (Fig. 1a). A typical FTIR-ATR spectrum of paraffin in comparison to characteristic virgin beeswax is presented in Fig. $1 \mathrm{~b}$. It is characterised by a simple molecular structure related to hydrocarbon absorption bands (at 2921, 2852, 1464 and $719 \mathrm{~cm}^{-1}$ ). The main spectral differences between pure beeswax and paraffin are observed in the fingerprint region. The analyte signals related to the ester and free fatty acids vibrations (at 1739, 1714 and $1172 \mathrm{~cm}^{-1}$ ) are not present in the IR spectrum of paraffin, and therefore, represent valuable and indicative spectroscopic data for detection of beeswax adulteration. Contrary to the lack of these particular signals in the fingerprint region of a paraffin spectrum, a characteristic IR spectrum of beef tallow is dominated by the strong intensive absorption bends that correspond to the esters vibrations, occurring at 1745 and 1158 $\mathrm{cm}^{-1}$. These signals are considerably stronger and characterised by the absorption maxima shifts in comparison to beeswax signals (Fig. 1b).

\section{Spectral features (FTIR-ATR) of beeswax mixtures with paraffin and tallow}

As presented in Fig. 2a, IR spectra of prepared virgin beeswax-paraffin mixtures containing different proportions of paraffin (5 - 95\%), revealed a clear
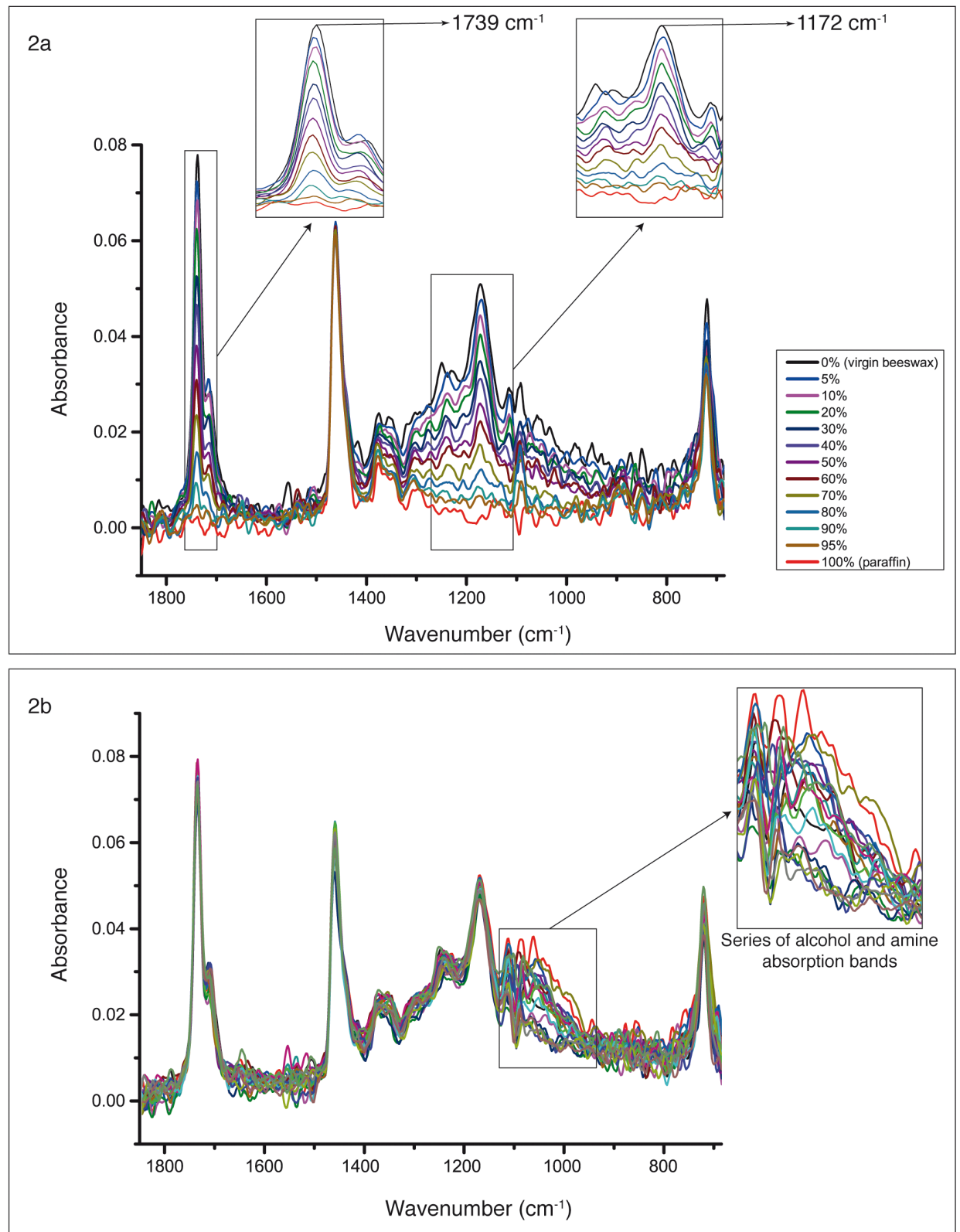

Fig. 2. (a) IR spectra of virgin beeswax, paraffin, and beeswax-paraffin mixtures containing different proportions of paraffin with an emphasis on indicative spectral regions; (b) Spectral variations between different virgin beeswax samples. 
linear decrease in the absorption intensities of the lipid components following the increasing percentages of the paraffin. These effects were observed in the spectral region between 1765 and $1700 \mathrm{~cm}^{-1}$ (esters and free fatty acids; absorption maximums at $1739 \mathrm{~cm}^{-1}$ and $1714 \mathrm{~cm}^{-1}$, respectively) and 1300 and $1130 \mathrm{~cm}^{-1}$ (esters; absorption maximum at $1172 \mathrm{~cm}^{-1}$ ).

When comparing the spectral variations identified in different virgin beeswax samples $(n=21)$ (Fig. 2b) with absorption changes observed in beeswaxparaffin mixtures, it is obvious that the spectral variations determined in different beeswaxes do not intercept those crucial for detection of beeswax adulteration with paraffin. As presented in Fig. 2b, the most variable part of a virgin beeswax spectra belongs to the absorption vibrations of alcohols and amines, observed in the spectral region between 1130 and $970 \mathrm{~cm}^{-1}$. Furthermore, no spectral differences between samples of genuine beeswax before and after the temperature treatment $\left(125^{\circ} \mathrm{C}\right.$, 45 minutes) were determined (results not shown). As presented in Fig. 3, the results showed a similar detection accuracy for the tallow adulteration level in beeswax-beef tallow mixtures and linear behaviour where IR absorption intensities (most prominent in the spectral ranges between 1745 $1739 \mathrm{~cm}^{-1}$ and $1172-1158 \mathrm{~cm}^{-1}$ ) increased linearly for the increasing percentages of the tallow added to beeswax (Fig. 3a).
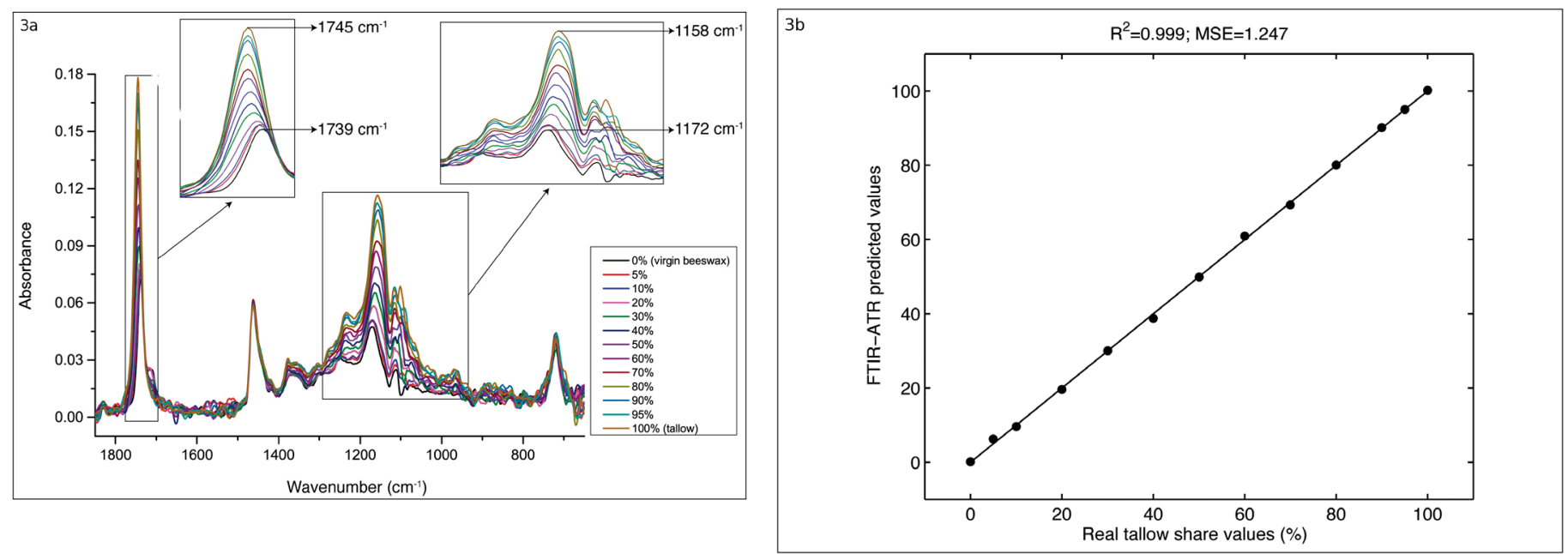

Fig. 3. (a) IR spectra of virgin beeswax, beef tallow, and beeswax-tallow mixtures (b) PLSR calibration plot of the real beeftallow share values versus FTIR-ATR predicted values.
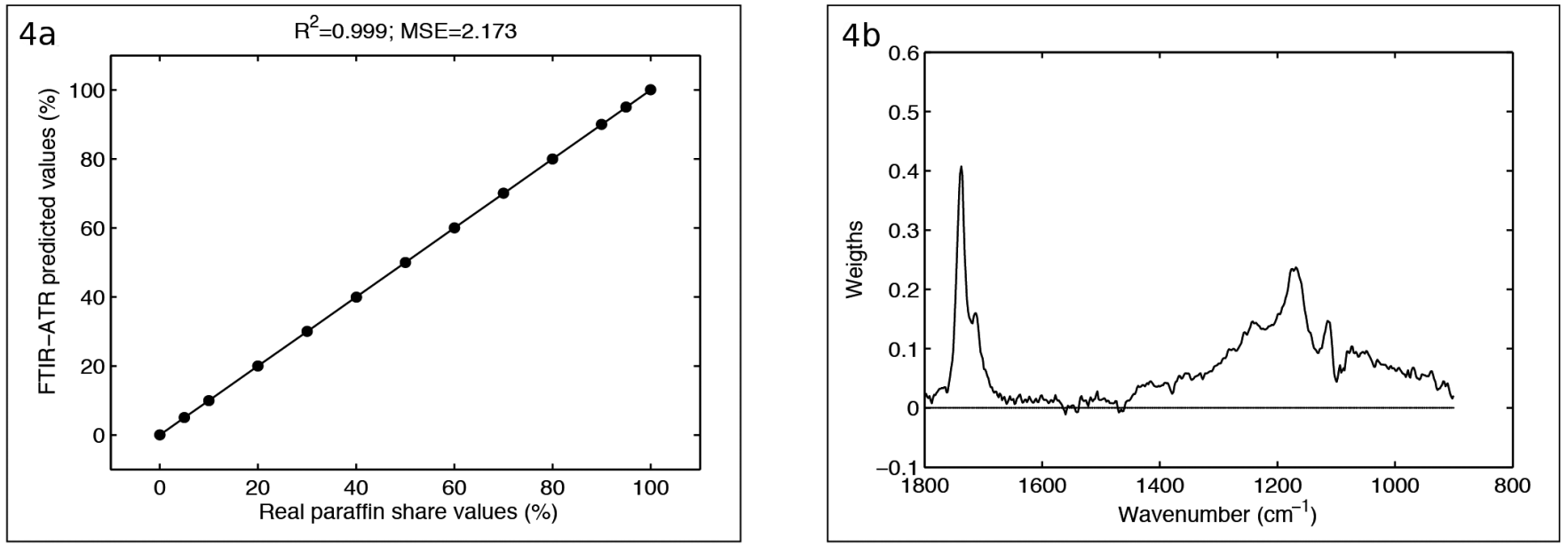

Fig. 4. Prediction performance parameters of PLSR model constructed for determination of the paraffin share in beeswax: (a) PLSR calibration plot of the real-paraffin share values versus FTIR-ATR predicted values; (b) Effects of the first PLS latent component. 


\section{J. APCL. SCL. VOL. 59 NO. 22015}

\section{Detection of adulterants in beeswax by FTIR-ATR spectroscopy}

Partial least squares regression (PLSR) calibration and model performance parameters of the paraffin share in adulterated mixtures predicted by IR spectral data are presented in Fig. 4a. The results obtained by using the fingerprint region have revealed a strong predictive strength $\left(R^{2}=0.999\right)$ and good calibration accuracy (MSE $=2.173$ ) of FTIR spectroscopy for detection of the paraffin share in beeswax. An investigation of PLS latent components revealed that most of the variance in the spectral data set is explained by the first 6 latent components. Most of the information (99.84\%) was captured in eigenvalues compressed in the first PLS component, which correspond to the alterations of esters $\left(\sim 1739 \mathrm{~cm}^{-1}\right.$ and $\left.1172 \mathrm{~cm}^{-1}\right)$ and free fatty acid $\left(\sim 1714 \mathrm{~cm}^{-1}\right)$ absorption bands (Fig. 4b). Partial least squares regression calibration and model performance parameters of the tallow share predicted by IR spectra (Fig. 3b) also revealed good predictive strength $\left(R^{2}=0.999\right)$ and calibration accuracy (MSE $=1.247)$.

A constructed PLSR model was used to predict the paraffin share in comb foundation sample set in which the samples were of an unknown chemical background. The results of the distribution of comb foundation samples according to the paraffin proportion predicted by the IR spectra, are presented in Fig. 5. As summarised and categorised in Tab. 1, the majority of the analysed samples (89\%) were adulterated with paraffin. Only 6 out of $56(11 \%)$ samples were identified as virgin beeswax, $28 \%$ of samples exhibited a higher level of paraffin adulteration ( $>46 \%$ of paraffin), while the majority of the analysed samples (50\%) were found to be adulterated with $5-20 \%$ of paraffin. The red line represents the limit-area that distinguishes genuine beeswax samples (below the red line) from adulterated ones. The results have revealed paraffin (and/or microcrystalline wax) as the only adulterants detected in the studied comb foundations. No other adulterant traces (no spectral features indicating the presence of other adulterants) were found in the collected comb foundations, based on the qualitative inspection of their IR spectra and comparison to IR spectra of the most common adulterants (personal spectral collection and the literature). These observations confirm the prevalence of paraffin adulteration on the beeswax market.

\section{Measurements of physico-chemical parameters used for detection of beeswax adulteration}

Descriptive statistics for analysed physico-chemical parameters for virgin beeswax and comb foundations collected from the international market is presented in Table 2. The results revealed anomalous values for particular virgin beeswax samples in which the values were out of the range officially proposed for pure beeswax. Higher acid number values (>22 mg $\mathrm{KOH} / \mathrm{g}$ ) were determined for 8 samples. Values of saponification and ester number outside the proposed ranges were found for 5 virgin beeswax samples, reaching the maximum value of 134 and $112.2 \mathrm{mg}$ $\mathrm{KOH} / \mathrm{g}$, respectively.

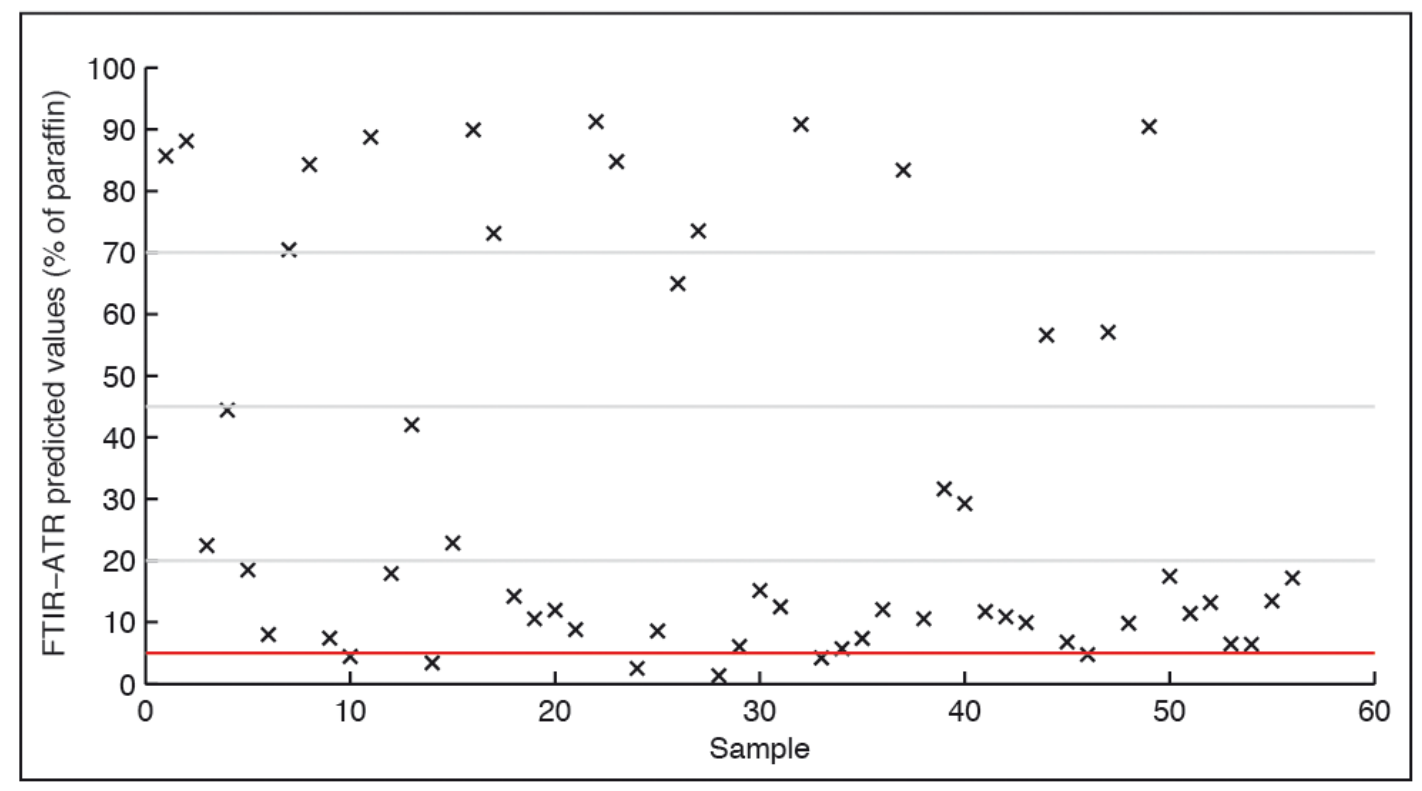

Fig. 5. PLSR prediction of the paraffin share in comb foundation samples $(n=56)$ using FTIR-ATR spectra with the fingerprint region $(1800-900 \mathrm{~cm}-1)$. 
The results showing the correlation between the values of the analysed physico-chemical parameters and paraffin share in beeswax-paraffin mixtures are presented in Fig. 6. It is clear from the scatter plot presented in Figure 6a that as the paraffin share increases, the acid value tends to decrease (negative correlation). This strong linear correlation between acid number and paraffin share indicates a good predictive strength of acid number for paraffin share $\left(R^{2}=0.989\right)$ in beeswax-paraffin mixtures. However, the changes of other physico-chemical values as the paraffin share increases did not follow the linear pattern well. Ester number $\left(R^{2}=0.788\right)$ and saponification number $\left(R^{2}=0.883\right)$ showed a moderate linear correlation with the paraffin share, as presented in Figure $6 \mathrm{~b}$ and $6 c$, respectively. On the other hand, the ester/acid ratio $\left(R^{2}=0.611\right)$ and melting point $\left(R^{2}=0.564\right)$ exhibited almost no linear correlation with significant deviations (Fig. 6d and 6e).

The relationship between the prediction of the paraffin share in comb foundation samples by IR spectral data and by acid number (as the only parameter showing consistent results with respect to paraffin share detection) is presented in Figure 7. It is obvious that the results obtained by two predictors are not congruent in paraffin share determination. The linear fit of IR spectral data for the paraffin proportion proved to be more accurate (Fig. 4a) in comparison to acid number prediction performance (Fig. 6a). Thus, it can be concluded that such a discrepancy is related to the acid number anomalies.

\section{DISCUSSION}

Infrared (IR) spectroscopy permits acquisition of the absorption spectra of compounds that are a unique reflection of their molecular structure. An infrared spectrum of a compound provides a unique "fingerprint" which is readily distinguished from the absorption patterns of all other compounds and it is, therefore, a valuable tool for identification and analysis of different substances in organic specimens. The method and observations reported by Maia et al. (2013) provided a valuable foundation and guidelines for further research; establishment of calibration curves (covering the entire concentration range of adulteration: 0 - 100\%) and validation of analytical procedure on unknown samples, which was performed in this study. The entire fingerprint region (1800 - $900 \mathrm{~cm}^{-1}$ ) comprising the crucial absorbance intensity changes (at 1739, 1714 and $1172 \mathrm{~cm}^{-1}$ ) was used in this study for chemometric modelling. In comparison to the investigation approach demonstrated in the feasibility study by

Table 1.

Distribution of analysed comb foundation samples ( $n=56$ ) by adulteration category

\begin{tabular}{cccc}
\hline Adulteration category & Paraffin share & n of samples & $\%$ of samples \\
\hline High level & $>71 \%$ & 12 & 21 \\
\hline Higher middle level & $46-70 \%$ & 4 & 7 \\
\hline Lower middle level & $21-45 \%$ & 6 & 11 \\
\hline Low level & $5-20 \%$ & 28 & 50 \\
\hline Genuine beeswax & $<5 \%$ & 6 & 11 \\
\hline
\end{tabular}

Table 2.

Physico-chemical parameters determined in virgin beeswax and comb foundation from the international market

\begin{tabular}{|c|c|c|c|c|c|c|c|}
\hline Sample & Physico-chemical parameter & $\bar{n}$ & Min & Max & Mean & SD & CV \\
\hline \multirow{5}{*}{ 高 } & Melting point $\left({ }^{\circ} \mathrm{C}\right)$ & 17 & 60 & 65 & 62.74 & 1.64 & 0.03 \\
\hline & Acid number (mg KOH/g) & 15 & 20.70 & 30.20 & 25.74 & 3.88 & 0.15 \\
\hline & Ester number $(\mathrm{mg} \mathrm{KOH} / \mathrm{g})$ & 11 & 31.10 & 112.20 & 74.46 & 23.13 & 0.31 \\
\hline & Ester/acid ratio & 11 & 1.18 & 5.14 & 3.01 & 1.11 & 0.37 \\
\hline & Saponification number (mg KOH/g) & 11 & 57.50 & 134.00 & 99.45 & 22.12 & 0.22 \\
\hline \multirow{5}{*}{ 号 } & Melting point $\left({ }^{\circ} \mathrm{C}\right)$ & 55 & 48 & 64 & 57.23 & 3.97 & 0.07 \\
\hline & Acid number (mg KOH/g) & 55 & 0.86 & 25.80 & 16.04 & 8.05 & 0.50 \\
\hline & Ester number (mg KOH/g) & 53 & 1.40 & 92.50 & 48.89 & 26.39 & 0.54 \\
\hline & Ester/acid ratio & 53 & 0.46 & 4.95 & 2.92 & 0.94 & 0.32 \\
\hline & Saponification number (mg KOH/g) & 53 & 4.20 & 117.70 & 65.24 & 33.80 & 0.52 \\
\hline
\end{tabular}



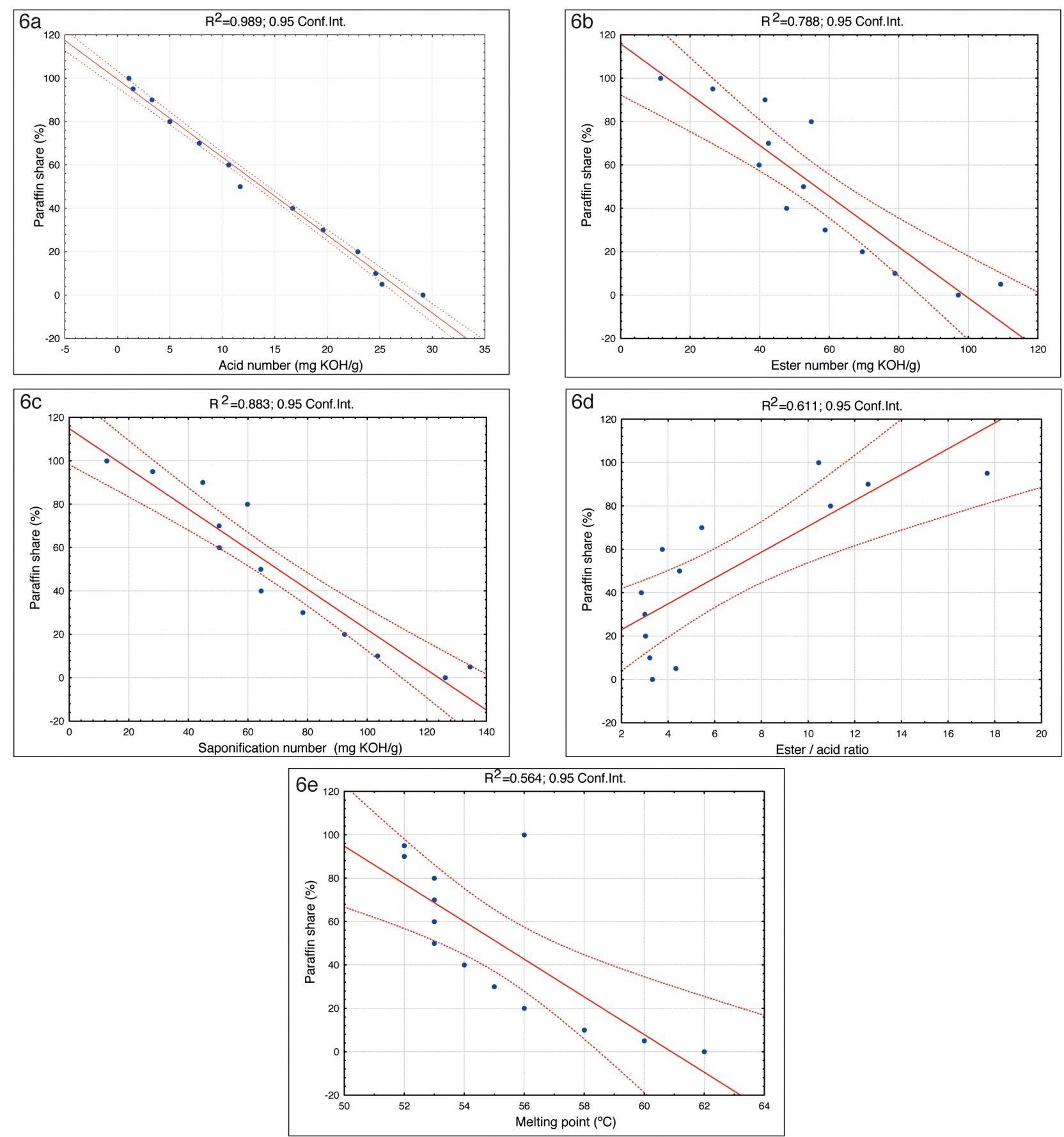

Fig. 6. Simple regression scatter plots showing the relationship between the values of physico-chemical measurands and the paraffin share in mixtures: (a) Acid number; (b) Ester number; (c) Saponification number; (d) Ester/acid ratio; (e) Melting point.

Maia et al. (2013) that involved the development of a quality index based on the calculation of chosen absorbance ratios $\left(I_{1739 \mathrm{~cm}-1} / I_{2852 \mathrm{~cm}-1}, I_{1714 \mathrm{~cm}-1} / I_{2852}\right.$ $\mathrm{cm}-1$ and $\left.I_{1739 \mathrm{~cm}-1} / I_{1714 \mathrm{~cm}-1}\right)$, a different spectral data processing approach was performed in this study. We used this approach to make the analytical procedure simpler, faster, and applicable for routine purposes. It was observed in this study that adulteration of beeswax with paraffin and microcrystalline wax could not be distinguished by FTIR-ATR. These findings were reported by Maia et al. (2013) as well. It can be assumed that such effects appear due to similar spectral features arising from the same petroleum-based origin (both waxes are made of long-chain alkane hydrocarbons; microcrystalline wax is additionally characterised by very fine crystalline structure). Given that the price of microcrystalline wax is almost the same as beeswax price, it 


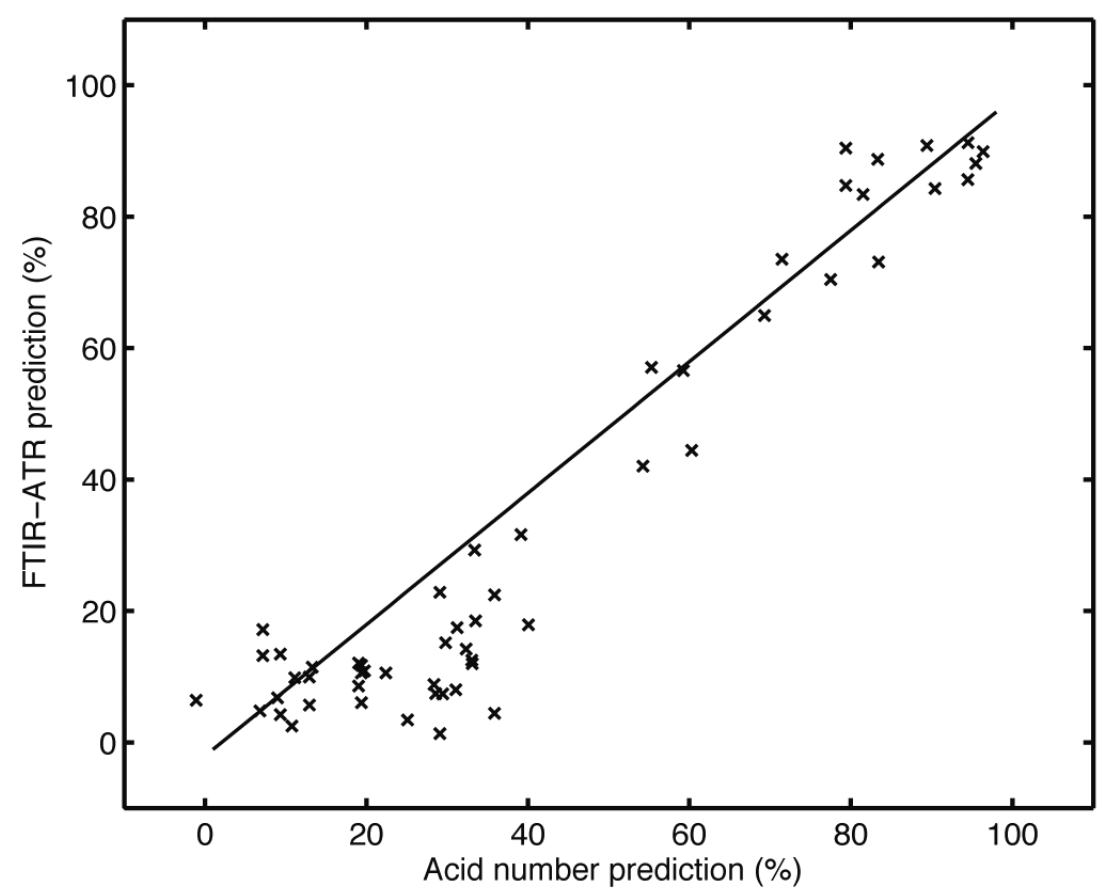

Fig. 7. Comparison of the paraffin share prediction performance by FTIR-ATR spectral data and acid number for comb foundation samples.

is unlikely from the economical point of view that beeswax is being adulterated with a substance of equal price to a larger degree. Thus, it can be assumed that paraffin prevails as a beeswax adulterant due to its significantly lower price. As far as adulteration goes, though, it is irrelevant which substance is added to beeswax since both substances represent contaminants that should not be present in beeswax. The spectral analysis of genuine beeswax samples revealed that no particular spectral differences appear when comparing white and yellow beeswax samples. Beeswax colour is mostly related to the fat-soluble carotenoid pigments that originate from pollen, but various undefined contaminants that are collected and absorbed in the beeswax over time may also affect the colour (Winston, 1991; Berry and Delaplane, 2001). These substances appear in beeswax in traces, so one can assume that they provide little or no IR information. It could also be that the signals of these substances are overlapped by more prominent absorption bands, and thus, do not compromise chemical analyte signals favourable for adulteration detection.

According to the prediction of the paraffin share in comb foundation samples based on their IR spectral data, 6 samples were placed below the $5 \%$ detection limit reported for paraffin. It can be assumed, that the paraffin share that was less than $5 \%$ was tentatively detected in those samples due to minor absorbance variations that might occur among different virgin beeswax samples in the spectral region indicative for paraffin detection. The samples exhibiting less than a $5 \%$ share of paraffin were, therefore, identified as genuine beeswax. The mean squared error (MSE) value (2.173\%) calculated in this study and the $5 \%$ detection limit determined for paraffin in previous spectroscopic findings (Maia et al., 2013), were in this regard taken into account. The quality criteria for routine beeswax testing proposed by the IHC (International Honey Commission), defined range values for 10 physicochemical parameters. Values of saponification and ester number outside the proposed range-values (87 - $102 \mathrm{mg} \mathrm{KOH} / \mathrm{g}$ and $70-90 \mathrm{mg} \mathrm{KOH} / \mathrm{g}$, respectively) were determined in this study for 5 virgin beeswax samples, which resulted in anomalous minimum and maximum values. Additionally, higher acid number values (>22 mg KOH/g) were also observed for 8 samples. Nevertheless, the same samples did not show deviations in their IR spectra; they exhibited spectrum characteristic for pure beeswax. The beeswax samples used in this study were definitely authentic (collected directly from the experimental beehives, containing no unnatural residues). Thus, the results suggest that the definition and identification of pure beeswax by currently proposed physico-chemical values is not reliable and may lead to a wrong interpretation of a sample's authenticity. As expected, values of the proposed physico-chemical parameters obtained for 
comb foundation revealed even a higher degree of inconsistencies and anomalous values. Similar results were reported by Bernal et al. (2005) who investigated the influence of the relative amounts (2 - 50\%) of adulterants (paraffin, stearic acid, animal tallow, and carnauba wax) on the values of the commonly used physico-chemical parameters. The results have revealed inconsistency and a frequent appearance of anomalous values for the majority of the analysed parameters.

Furthermore, the reliability of the proposed analytical values for pure beeswax characterisation and detection of adulteration is jeopardised by the temperature treatment $>100^{\circ} \mathrm{C}$ which can cause a significant increase in ester value and decreases in acid value (Tulloch, 1973; Bogdanov, 2009). Such effects inevitably occur in practice (comb foundation production process) given that beeswax is commonly being subjected to different high temperature treatments $\left(121-140^{\circ} \mathrm{C}\right)$ necessary to kill the spores of the heat-resistant Paenibacillus larvae larvae.

The results of this research revealed disturbing findings; $89 \%$ of comb foundation samples collected from the market were found to be adulterated with paraffin. We believe that this kind of outcome is the consequence of the larger-scale problem of a general deficit of beeswax and a "chronic" accumulation and circulation of the paraffin in the comb foundation production process. The lack of routine analytical tools, and regulations controlling the beeswax origin or any kind of its monitoring, definitely contribute to this issue. The chemical composition of beeswax is generally not being controlled routinely, and therefore, allows continuous and successive contamination of beeswax by adulterants, primarily paraffin. Similarly to the results obtained in this study, a high percentage of comb foundation samples collected from the Portuguese market $(60 \%)$ were adulterated with paraffin/microcrystalline wax, as reported by Maia et al. (2013). The disconcerting situation on the comb foundation market, indicated by current and previous studies, implies an urgent need for routine beeswax authenticity control.

The objective and results presented in this study fit the current objective of the IHC; to work out standards for other bee products besides honey, including the development of better and new analysis methods. The FTIR-ATR analytical procedure and adulteration-level categorisation, established in this study for distinguishing pure from adulterated beeswax, can be used as a guideline to further upgrade of this method. But verification should be performed on alarger data set of both virgin beeswax and different beeswax adulterant types. To the best of our knowledge, no method with a calibration set covering the entire concentration range of paraffin and tallow adulteration in beeswax was employed until now. It was a crucial step prior to the establishment of a protocol that provides accurate determination of the share of adulterants in beeswax.

It can be assumed, that adulterated beeswax foundation can cause economic losses in beekeeping. It was reported that adulterated beeswax foundation may have an impact on brood rearing. The results can be abnormalities and disorders of brood development, and consequently, weakness and increased mortality of honeybee colonies (Wallner, 2005). Frequent reports on deformed or completely destroyed combs by the Croatian beekeepers (personal communication) indicate the above described effects might be possible. An investigation on this subject was recently launched by Semkiw and Skubida (2013), investigating the comb construction and brood development on beeswax foundation adulterated with paraffin. The results have revealed that paraffin used in their experiment had no negative impact on the bee colonies. However, the authors emphasized that it is impossible to exclude the possibility that paraffin of different chemical compositions and purities may cause harmful effects on bees.

We believe that the fact that adulteration of any product represents deliberate destruction of the product's quality aiming to achieve an illegal profit and deceive consumers / beekeepers, along with indications on negative effects of adulterated foundations on honeybee colonies, should trigger the general initiative for routine monitoring of the authenticity of the beeswax comb foundations present on the market.

\section{CONCLUSIONS}

In this study we demonstrated that an analytical approach defining the standard curves for particular adulteration levels (5 - 95\%) based on chemometric modelling of specific spectral data set indicative for beeswax adulteration (fingerprint region), allows the same contamination levels to be determined for unknown samples (comb foundations).

The PLS regression model performance parameters showed the strong predictive power of FTIR-ATR spectroscopy for predicting the share of paraffin and tallow in beeswax $\left(R^{2}=0.999\right)$. The predictive power indicates the analytical reliability of this method for routine quality control. Moreover, good 
calibration performance and detection accuracy for the paraffin and tallow adulteration levels initially investigated in this study, strongly suggest the possibility of developing the same analytical protocol for other commonly used beeswax adulterants. On the other hand, unsatisfactory regression performance parameters obtained for the correlation investigation between the values of the studied physicochemical parameters and paraffin share in mixtures, confirmed the unsuitability of existing physicochemical analytical tools for reliable detection of adulterants in beeswax (with the exception of acid number).

Besides providing results that are accurate, consistent, comparable, and within acceptable limits of precision, the advantages of the demonstrated method over commonly used methods are that it is fast, reliable, cheap, and easy-to-use. Also, FTIR-ATR measurements require no sample preparation (reagent-free method) and only a small amount of the sample ( 0.05g). These factors further support the applicability of the proposed FTIR-ATR analytical procedure for routine beeswax analysis and quality control.

\section{ACNOWLEDGMENTS}

This work has been supported in part by the Croatian Science Foundation under the project NaPro-Flav (8547). The research was also supported by the Paying Agency for Agriculture, Fisheries and Rural Development and VIP Project No. 2013-12-62. The support has been greatly appreciated. The authors also thank the Croatian Beekeepers Association and Mr. Predrag Manger for the sampling support.

\section{REFERENCES}

Aichholz R., Lorbeer E. (1999) Investigation of combwax of honeybees with high-temperature gas chromatography and high-temperature gas chromatography-chemical ionization mass spectrometry I. High-temperature gas chromatography. Journal of Chromatography A 855(2): 601-615. DOl: 10.1016/S0021-9673(99)00725-6

Aichholz R., Lorbeer E. (2000) Investigation of combwax of honeybees with high-temperature gas chromatography and high-temperature gas chromatography-chemical ionization mass spectrometry. Il: High-temperature gas chromatography-chemical ionization mass spectrometry. Journal of Chromatography A 883(1-2): 75-88. D0l: 10.1016/S0021-9673(00)00386-1
Bernal J. L., Jiménez J. J., del Nozal M. J., Toribio L., Martín M. T. (2005) Physico-chemical parameters for the characterization of pure beeswax and detection of adulterations. European Journal of Lipid Science and Technology 107(3): 158-166. DOl: 10.1002/ejlt.200401 105

Berry A. J., Delaplane S. K. (2001) Effects of comb age on honey bee colony growth and brood survivorship. Journal of Apicultural Research 40(1): 3-8. D0l: 10.1002/ ejlt.200401 105

Beverly M. B., Kay P. T., Voorhees K. J. (1995) Principal component analysis of the pyrolysis mass spectra from African, Africanized hybrid and European beeswax. Journal of Analytical and Applied Pyrolysis 34(2): 251-263. DOl: 10.1016/0165-2370(95)00891-H

Birshtein V. Y, Tul'chinskii V. M. (1977) Determination of beeswax and some impurities by IR spectroscopy. Chemistry of Natural Compounds 13(2): 232-235.

Bogdanov S. (2004a) Beeswax: quality issues today. Bee World 85(3): 46-50.

Bogdanov S. (2004b) Quality and standards of pollen and beeswax. Apiacta 38: 334-341.

Bogdanov S. (2009) Beeswax: Production, properties, composition and control. Beeswax book. Bee Product Science. 17 pp.

Breed M. D., Garry M. F., Pearce A. N., Hibbard B. E., Bjostad L. B., Page R. E. (1995) The role of wax comb in honeybee nestmate recognition. Animal Behaviour 50(2): 489496. DOl: 10.1006/anbe.1995.0263

Buchwald R., Breed M. D., Bjostad L., Hibbard B. E., Greenberg A. R. (2009) The role of fatty acids in the mechanical properties of beeswax. Apidologie 40(5): 585-594. DOl: 10.1051/apido/2009035

D'Ettorre P." Wenseleers T., Dawson J., Hutchinson S. Boswell T., Ratnieks F. L. W. (2006) Wax combs mediate nestmate recognition by guard honeybees. Animal Behaviour 71(4): 773-779. DOl: 10.1016/j.anbehav.2005.05.014

DGF-M-V-6 (1 957) DGF - Einheitsmethoden - Abteilung M - Wachse. German Standard -Beeswax. 29 pp. Available at: http://www.dgfett.de/methods/inhaltsverzeichnis.pdf 
Edwards H. G. M., Farwel D. W., Daffner L. (1996) Fouriertransform Raman spectroscopic study of natural waxes and resins. Spectrochimica Acta Part A: Molecular and Biomolecular Spectroscopy 52(12): 1639-1648. DOl: 10.1016/0584-8539(96)01730-8

Council of Europe (2007) European Pharmacopoeia. $6^{\text {th }}$ Edition. Council of Europe. Strasbourg, Cedex, France. Volume 2. 1085-3308 pp.

Fröhlich B., Riederer M., Tautz J. (2000) Comb-wax discrimination by honeybees tested with the proboscis extension reflex. Journal of Experimental Biology 203(10): $1581-1587$.

Jiménez J. J., Bernal J. L., Aumente S., Toribio L., Bernal J. (2003) Quality assurance of commercial beeswax II. Gas chromatography-electron impact ionization mass spectrometry of alcohols and acids. Journal of Chromatography A 1007(1-2): 101-1 16. DOl: 10.1016/S00219673(03)00962-2

Jiménez J. J., Bernal J. L., del Nozal M. J., Martín M. T., Bernal J. (2006) Sample preparation methods for beeswax characterization by gas chromatography with flame ionization detection. Journal of Chromatography A 1129(2): 262-272. DOl: 10.1016/j.chroma.2006.06.098

Jiménez J. J., Bernal J. L., del Nozal M. J., Martín T., Toribio L. (2009) Identification of adulterants added to beeswax: Estimation of detectable minimum percentages. European Journal of Lipid Science and Technology 111 (9): 902-91 1. DOl: 10.1002/ejt.200800263

Jiménez J. J., Bernal J. L., del Nozal M. J., Toribio L., Bernal J. (2007) Detection of beeswax adulterations using concentration guide-values. European Journal of Lipid Science and Technology 109(7): 682-690. DOl: 10.1002/ ejlt.200600308

Jiménez J. J., Bernal J. L., Aumente S., del Nozal M. J., Martín M. T., Bernal J. (2004) Quality assurance of commercial beeswax. I. Gas chromatography- electron impact ionization mass spectrometry of hydrocarbons and monoesters. Journal of Chromatography A 1024 (1-2): 147154. DOI: 10.1016/j.chroma.2003.10.063

Maia M., Nunes F. M. (2013) Authentication of beeswax (Apis mellifera) by high-temperature gas chromatography and chemometric analysis. Food Chemistry 136(2): 961-968. DOl: 10.1016/j.foodchem.2012.09.003
Maia M., Barros A. R. N. A., Nunes F. M. (2013) A novel, direct, reagent-free method for the detection of beeswax adulteration by single-reflection attenuated total reflectance mid-infrared spectroscopy. Talanta 107: 74 80. DOl: 10.1016/j.talanta.2012.09.052

MatLab 7.11 (2010b) MathWorks Inc.

Muscat D., Tobin M. J., Guo Q., Adhikari B. (2014) Understanding the distribution of natural wax in starch-wax films using synchrotron-based FTIR (S-FTIR). Carbohydrate Polymers 102: 125-135. DOl: 10.1016/j.carbpol.2013.1 1.004

Semkiw P." Skubida P. (2013) Comb construction and brood development on beeswax foundation adulterated with paraffin. Journal of Apicultural Science 57(1):75-83. DOl: 10.2478/jas-2013-0009

Serra Bonvehi J. S., Orantes Bermejo F. J. (2012) Detection of adulterated commercial Spanish beeswax. Food Chemistry 132(1): 642-648. DOl: 10.1016/j.foodchem.201 1.10.104

Statistica ver. 7. (2004) StatSoft Inc.

Tulloch A. P. (1973) Factors affecting analytical values of beeswax and detection of adulteration. Journal of the American Oil Chemists Society 50(7): 269-272.

University of Tartu (Estonia), Institute of Chemistry. Database of ATR-IR spectra of materials related to paints and coatings. Available at: http://tera.chem.ut.ee/IR_spectra/

Wallner K. (2005) Foundation causing honeybee brood damage. In: Proceedings of ICPBR 9. International Symposium. York - United Kingdom. 12-14 October 2005: 30.

Waś E., Szczęsna T., Rybak-Chmielewska H. (2014) Determination of beeswax hydrocarbons by gas chromatography with a mass detector (GC-MS) technique. Journal of Apicultural Science 58(1): 145-157. DOl: 10.2478/JAS2014-0015

Winston M. L. (1991) The Biology of the Honey Bee. Harvard University Press. Cambridge. 36 pp.

Zimnicka B., Hacura A. (2006) An Investigation of Molecular Structure and Dynamics of Crude Beeswax by Vibrational Spectroscopy. Polish Journal of Environmental Studies 15(4A): 112-114. 